\title{
Generation of Perfect Optical Vortices by Using a Transmission Liquid Crystal Spatial Light Modulator
}

\author{
Nelson Anaya Carvajal, Cristian H. Acevedo, and Yezid Torres Moreno \\ GOTS, Grupo de Óptica y Tratamiento de Señales, Escuela de Física, Universidad Industrial de Santander, \\ Carrera 27 Calle 9, A.A. 678, 680002 Bucaramanga, Colombia
}

Correspondence should be addressed to Cristian H. Acevedo; cristian_rvd@yahoo.com

Received 21 November 2016; Revised 22 January 2017; Accepted 20 February 2017; Published 30 March 2017

Academic Editor: Stefan Wabnitz

Copyright (C) 2017 Nelson Anaya Carvajal et al. This is an open access article distributed under the Creative Commons Attribution License, which permits unrestricted use, distribution, and reproduction in any medium, provided the original work is properly cited.

\begin{abstract}
We have experimentally created perfect optical vortices by the Fourier transformation of holographic masks with combination of axicons and spiral functions, which are displayed on a transmission liquid crystal spatial light modulator. We showed theoretically that the size of the annular vortex in the Fourier plane is independent of the spiral phase topological charge but it is dependent on the axicon. We also studied numerically and experimentally the free space diffraction of a perfect optical vortex after the Fourier back plane and we found that the size of the intensity pattern of a perfect optical vortex depends on the topological charge and the propagation distance.
\end{abstract}

\section{Introduction}

As is well known, an optical vortex beam is an electromagnetic wave with a helical wavefront due to phase singularities [1]. These phase singularities are threads of darkness embedded within light fields in their spatial distribution, points for $2 \mathrm{D}$, and lines for $3 \mathrm{D}[2,3]$. Allen and his collaborators proved that the complex amplitude of an optical vortex possessing an azimuthal phase factor $\exp (i m \theta)$ carries an orbital angular momentum of $\mathrm{m} \hbar$, where $m$ is the topological charge and $\theta$ is the azimuthal angle [4]. The unique optical properties of the optical vortices have been widely used in applications such as optical tweezers [5-8], image processing [9-11], communication systems in free space [12-14], and optical fibers $[15,16]$. Motivated by these applications several methods for generating the optical vortex beam have been proposed [1727]; however the diameter of these optical vortices is related to their topological charges. This property causes difficulties to achieve a high spatial accuracy and high orbital angular momentum coupling optical vortices into a fiber.

To solve these requirements, Ostrovsk et al. have introduced the perfect optical vortex (POV) concept [28]. The perfect optical vortices are electromagnetic waves whose ringwidth size and average ring-diameter (the arithmetic average of the inner and outer ring-diameters) are both independent of the topological charge. To experimentally generate the POVs or POV array a Gaussian beam (or a wave plane) is directed toward a special phase mask [28, 29], an axicon [30], or a phase mask by combining an axicon and a spiral phase function $[31,32]$. The modulation of these phase masks is programmed onto a reflection liquid crystal spatial light modulator (RLC-SLM) working in phase only mode. The masks are created with the discretization of the phase into $N$ levels depending on the characteristics of the RLC-SLM. The most of RLC-SLMs operate with an 8-bit dynamic range or 256 phase levels.

In this work, we present an experimental approach for generating perfect optical vortices by using of phase masks of three levels with shape of axicon and spiral phase functions, which are displayed in a transmission liquid crystal spatial light modulator. The intensity and vorticity of the POVs are measured with a CMOS camera and the far-field diffraction pattern through an equilateral triangular slit, respectively. We showed numerically and experimentally that the free space propagation diffraction patterns of the POVs are dependent on the distance and they are variant to ring-width size and to average ring-diameter. 


\section{Theory}

As mentioned earlier, perfect optical vortices are beams whose diameter and width-ring are independent of its topological charge. POVs can be approximately generated by means of the Fourier transformation from Bessel-Gauss (BG) beams [31]. The complex field amplitude of a BG beam with amplitude unit can be described in cylindrical coordinates $(\rho, \theta)$, as $[33]$

$$
U(\rho, \theta)=J_{m}\left(k_{r} \rho\right) e^{i m \theta} e^{-\rho^{2} / w_{o}^{2}},
$$

where $w_{o}$ is the waist width of the Gaussian beam, $k_{r}$ is the radial wave vector, and $J_{m}(\cdot)$ is an $m$ th order Bessel function of first kind.

The field of a POV is obtained in the back focal plane of a converging lens by substituting (1) in the Fourier transform diffraction spectrum yielding the result

$$
\begin{aligned}
U(r, \phi)= & \frac{k}{i 2 \pi f} \int_{0}^{\infty} J_{m}\left(k_{r} \rho\right) e^{-\rho^{2} / w_{o}^{2}} \\
& \cdot r d r\left(\int_{0}^{2 \pi} e^{-i k p r \cos (\phi-\theta) / f} e^{i m \theta} d \theta\right),
\end{aligned}
$$

where $f$ is the back focal length of the lens. By analytic solving of the integral in (2), we can obtain the Fourier diffraction spectrum for BG beams with different topological charges $m$ at the back focal plane of lens, which reads

$$
U(r, \phi)=i^{m-1} \frac{w_{o}}{w} I_{m}\left(\frac{2 r r_{o p}}{w^{2}}\right) e^{i m \phi} e^{-\left(r^{2}+r_{o p}^{2}\right) / w^{2}},
$$

with $r_{o p}$ and $w$, the radius and half width-ring of the perfect optical vortices, respectively. $I_{m}(\cdot)$ is an $m$ th order modified Bessel function of first kind and it can be written as [34]

$$
I_{m}(Z)=e^{-i m \pi / 2} J_{m}\left(Z e^{i \pi / 2}\right)
$$

The radius $r_{o p}$ in (3) can be expressed approximately as

$$
r_{o p}=\frac{k_{r} f}{k} \approx \frac{\lambda f}{P}
$$

where we had considered the experimental fact that $\sqrt{P^{2}+\lambda^{2}} \approx P$ (axicon period). It can see from (5) that the average radius of a POV is independent of the topological charge of the spiral phase and it basically governed Fourier transform of the axicon $[35,36]$. The columns three and four of Figure 1 provide simulations of the theoretical results for the intensity distribution of POVs with topological charges $m=1, m=2, m=3, m=5$, and $m=10$, obtained by Fourier transform diffraction of $\mathrm{BG}$ beams with axicon period $P=1.3[\mathrm{~mm}]$ and $P=0.7[\mathrm{~mm}]$, respectively. From Figure 1 , we can observe that the diameters of BG beams increase with the topological charge (first column) however; the diameters of POVs apparently do not change (third and fourth column). Also in Figure 1, one can see that the radii of the POVs of the fourth column are bigger than the radii of the POVs of the third column. Last result can be easily explained by decrease of the axicon period in (5) because $d$ is inverse to $r_{o p}$ The phase profiles of the BG beams on first column of Figure 1 are shown in second column of the same figure, while for the POVs on third and fourth column in Figure 1, the phase structures are equal and they are shown on fifth column of Figure 1. It is clear from each phase profile that the number of central dislocations is equal to the value of $m$ for each used $\mathrm{BG}$ beam and each generated POV.

Figure 2(a) illustrates the line profiles through the center of the intensity distributions of POVs shown in third column of Figure 1, which were obtained with an axicon period of $P=$ $1.3[\mathrm{~mm}]$. In this graph, we can observe a shift in hundredths of the millimeters among the ring-diameters for the POVs with the topological charges $m=1,2,3,5$ and $m=10$. This result can be explained by (3), in which the radial field amplitude of a POV depends on the combination of a Besselmodified function $I_{m}$ and a Gaussian function. Since the slope of the exponential function $I_{m}$ decreases slight as $m$ increases, the ring-diameter shifts by a small value when $I_{m}$ intersects the Gaussian function, as Figure 2(b) shows. However, the line profiles through the centers of the intensity distributions for the POVs with topological charges $m=1,2,3,5$ and $m=10$ have spatial shifts smaller than hundredths of the millimeters, when the POVs are generated with $P=0.7$ [mm], as shown in Figure 2(c). This behavior can be explained by taking into account the fact that the Bessel-modified functions for the POVs with $m=1,2,3,5$ and $m=10$ generated with $P=0.7[\mathrm{~mm}]$ have similar slopes, as shown in Figure 2(d). For this reason, taking this approximation on Fourier plane ( $w$ small), then for large $r_{o p}$, the function $I_{m}(\cdot)$ can written asymptotically as [35]

$$
I_{m}\left(\frac{2 r r_{o p}}{w^{2}}\right) \approx e^{2 r r_{o p} / w^{2}}
$$

Using (6), one finds easily the field distribution on the focal plane of the lens,

$$
U(r, \phi)=i^{m-1} \frac{w_{o}}{w} e^{i m \phi} e^{-\left(r+r_{o p}\right)^{2} / w^{2}} .
$$

So the intensity distribution of a POV can be calculated as

$$
I(r, \phi)=\left(\frac{w_{o}}{w}\right)^{2} e^{-2\left(\left(r+r_{o p}\right)^{2} / w^{2}\right)} .
$$

This result explains the fact that intensity pattern of a POV does not depend upon the topological charge when $r_{o p}$ is large and the axicon period is small.

On the other hand, we also can verify the nature of the POVs through their far-field diffraction patterns by an equilateral triangular aperture. These far-field diffraction patterns can be obtained considering a POV as a centered incident beam on an equilateral triangular aperture located at plane $z_{o}=0$. The size of the equilateral triangular aperture has been adjusted to POV size under consideration that the diameter of the bright ring does not depend on the topological charge $m$. Then the diffracted field of a POV by an equilateral triangular aperture is proportional to the Fourier transform, when it 


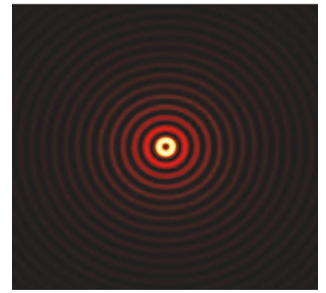

(a)

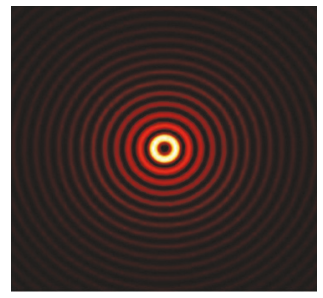

(b)

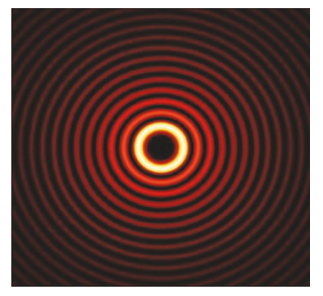

(c)

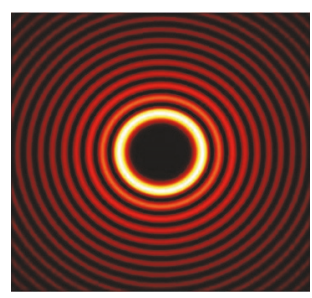

(d)

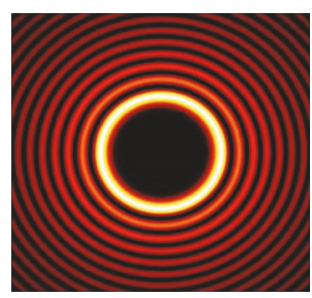

(e)

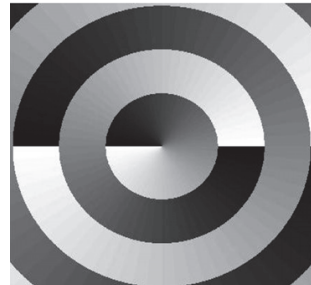

(f)

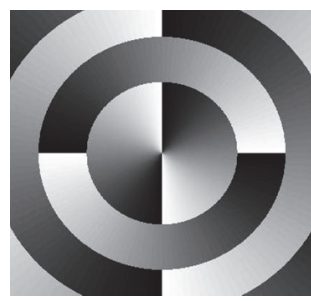

(g)

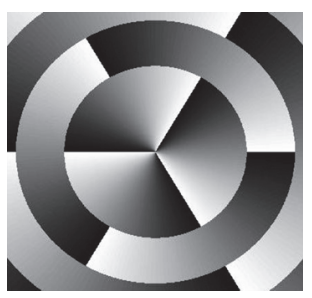

(h)

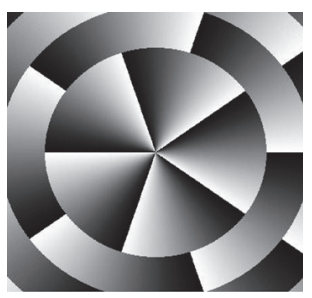

(i)

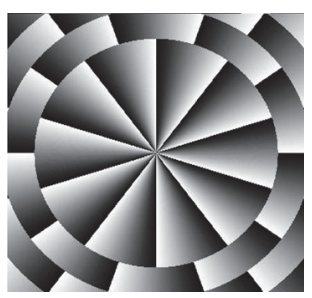

(j)

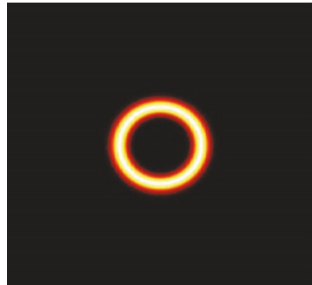

(k)

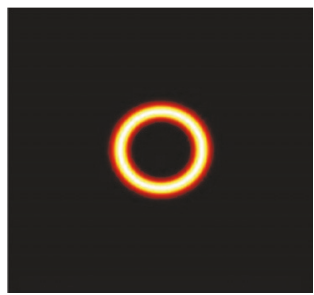

(1)

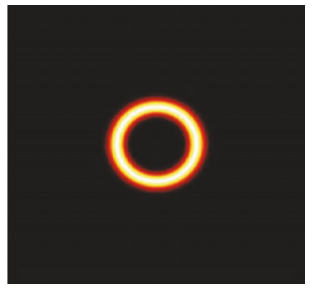

(m)

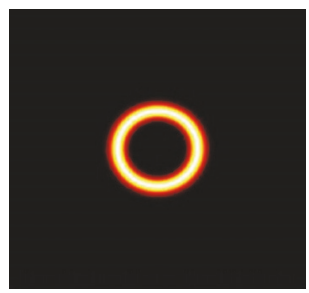

(n)

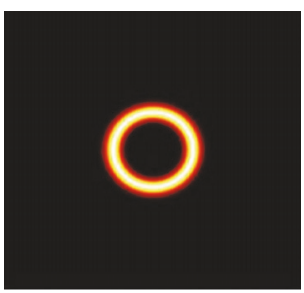

(o)

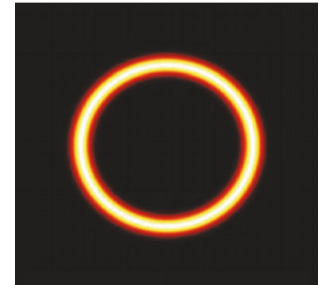

(p)

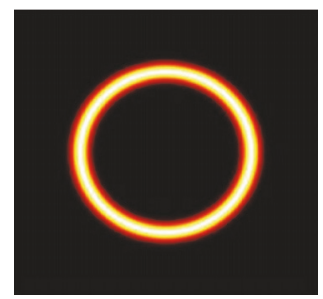

(q)

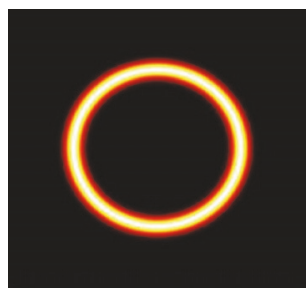

(r)

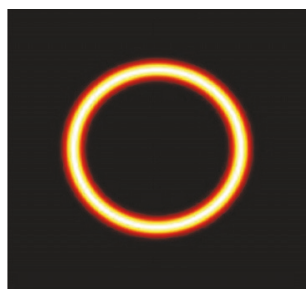

(s)

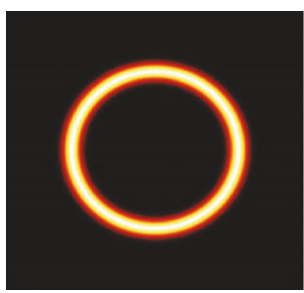

(t)

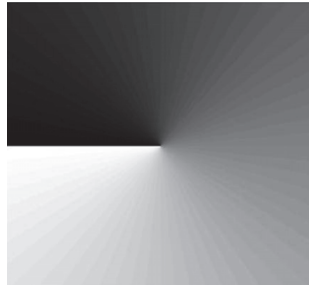

(u)

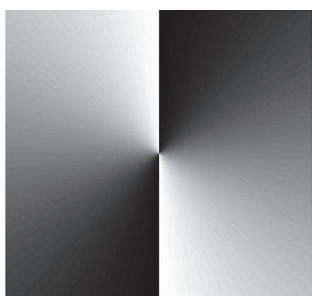

(v)

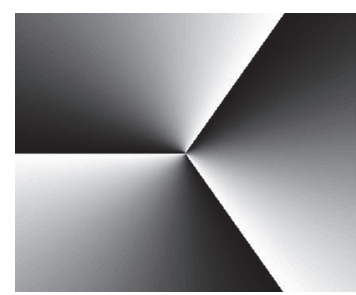

(x)

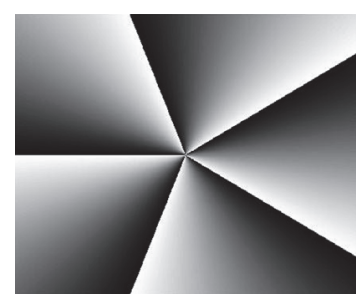

(w)

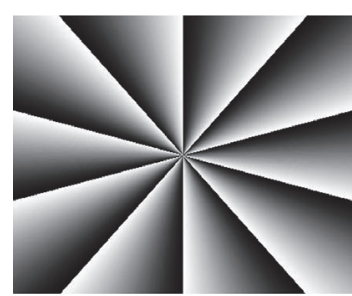

(y)

FIGURE 1: Simulations of the intensity patterns and the phase structures for Bessel-Gauss beams and perfect optical vortices. (a)-(e) Intensity distributions of the BG beams with topological charges $m=1,2,3,5$ and $m=10$, respectively. (k)-(o) and (p)-(t) Intensity distributions of POVs after the Fourier transformation of (a)-(e) for $P=1.3[\mathrm{~mm}]$ and $P=0.7[\mathrm{~mm}]$, respectively. (f)-(j) and (u)-(y) Phase profiles for the BG beams and the POVs, respectively. In the phase structures the black and white colors are corresponding to 0 and $2 \pi$. We used $\lambda=632.8$ $[\mathrm{nm}]$ and $f=380[\mathrm{~mm}]$ here and for theoretical and numerical solutions shown in Figures 2, 3, and 4. 


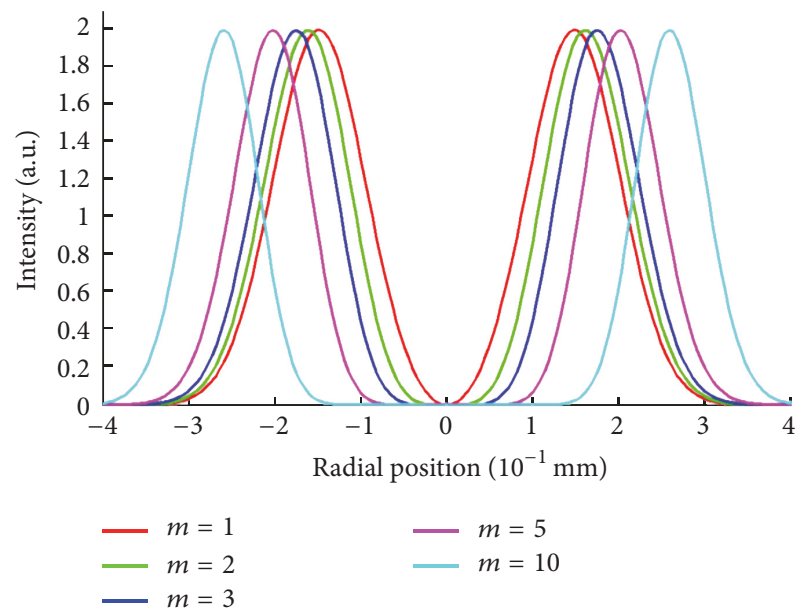

(a)

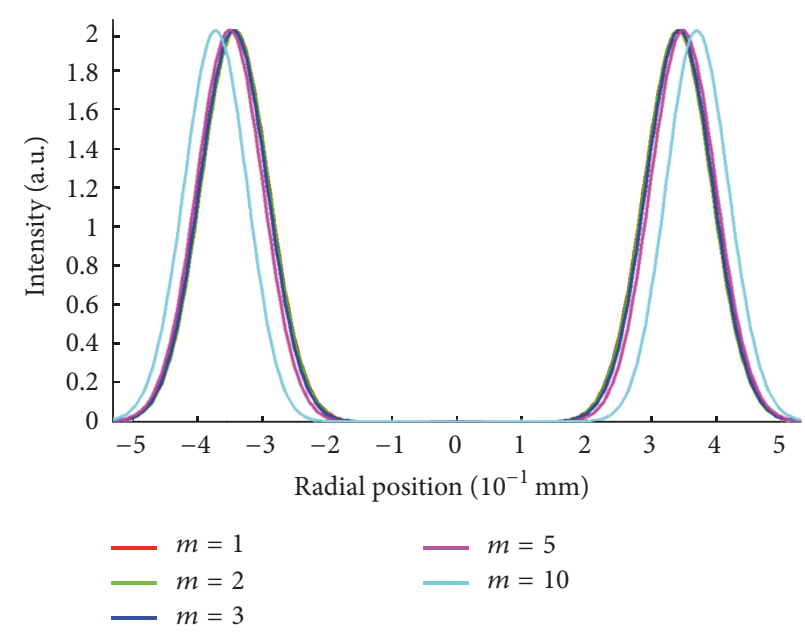

(c)

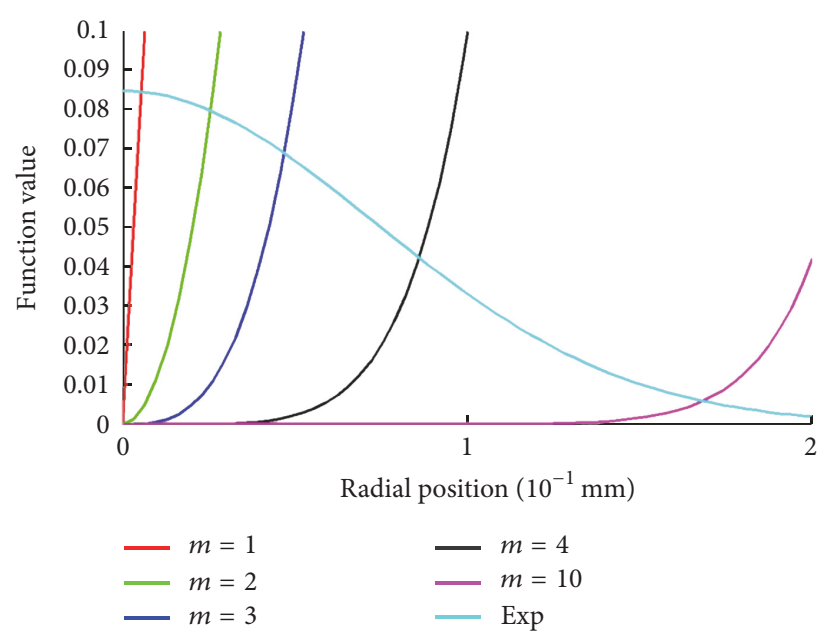

(b)

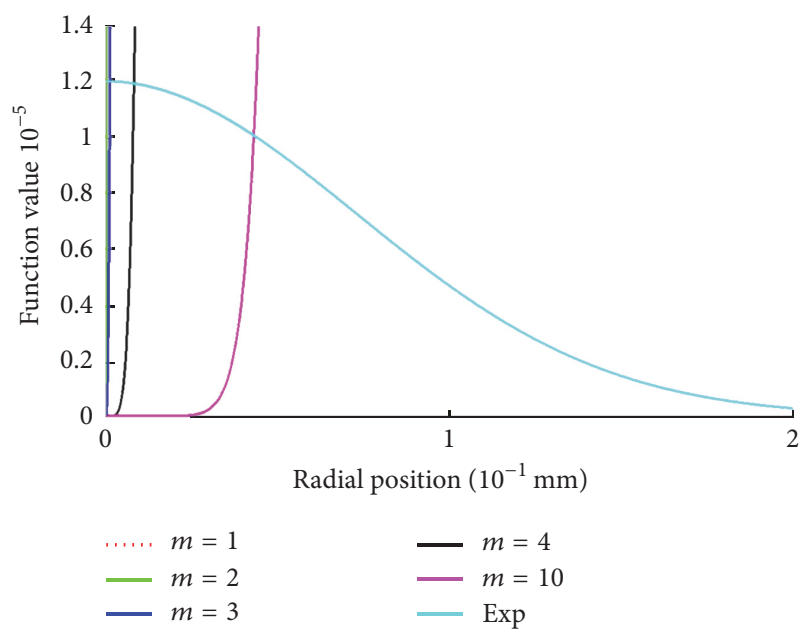

(d)

Figure 2: (a) and (c) Theoretical line intensity profiles along the vortex centers of POVs with topological charges $m=1,2,3,5$ and $m=10$, generated with $P=1.3[\mathrm{~mm}]$ and $P=0.7[\mathrm{~mm}]$, respectively. (b) and (d) Gaussian function and Bessel-modified functions for the topological charges $m=1,2,3,5,10$ versus radial distance; (b) and (d) were generated using $P=1.3[\mathrm{~mm}]$ and $P=0.7$ [mm], respectively. In (c) and (d), $m=1,2,3$ curves are a little bit different and cannot be distinguished by eye.

is used as standard $2 f^{\prime}$ Fourier transforming optical system based on a lens of focal length $f^{\prime}[37]$

$$
\begin{aligned}
& U\left(x_{o}^{\prime}, y_{o}^{\prime} z_{o}\right) \\
& \quad \propto \int_{-\infty}^{\infty} d x \int_{-\infty}^{\infty} U\left(x_{o}, y_{o}\right) t\left(x_{o}, y_{o}\right) e^{-i\left(x_{o}^{\prime} x_{o}+y_{o}^{\prime} y_{o}\right)},
\end{aligned}
$$

where $z_{0}=2 f^{\prime}, t\left(x_{o}, y_{0}\right)$ is the transmission function aperture which has unitary amplitude inside the aperture and zero, and $U\left(x_{o}, y_{o}\right)$ is the complex field of the POV in Cartesian coordinates $\left(x_{o}, y_{o}, z_{o}=0\right)$ written as

$$
\begin{aligned}
U\left(x_{o}, y_{o}\right)= & i^{m-1} \frac{w_{o}}{w} I_{m}\left(\frac{2 r_{o p} \sqrt{x_{o}^{2}+y_{o}^{2}}}{w^{2}}\right) \\
& \cdot e^{i m \tan ^{-1}\left(y_{o} / x_{o}\right)} e^{-\left(r^{2}+x_{o}^{2}+y_{o}^{2}\right) / w^{2}}
\end{aligned}
$$

By numerically solving the integral in (9), we can find the far-field intensity profile $\left|U\left(x_{o}^{\prime}, y_{o}^{\prime}, z_{o}\right)\right|^{2}$ for the POVs with different topological charges $m$. Figure 3 shows the POVs with topological charges, $m=1,2,3,5$, and 10 , and their respective numerical far-field diffraction pattern. On the diffraction pattern of POVs the formation of a truncated triangular lattice can be seen, in which the number of the spots along an edge of the triangle minus one represents the topological charge of the incident POV [38].

Finally, it is possible to analyze the comportment of a POV after the Fourier plane if it considers the Fresnel formula for the diffraction in free space given by [39]

$$
\begin{aligned}
& U\left(r_{1}, \phi_{1}, z\right)=\frac{k e^{i k z}}{i 2 \pi z} \\
& \cdot \int_{0}^{\infty} \int_{0}^{2 \pi} U(r, \phi) e^{i k\left(\left(r_{1}^{2}+r^{2}-2 r r_{1} \cos \left(\phi_{1}-\phi\right)\right) / 2 z\right)} \\
& \cdot r_{1} d r_{1} d \phi_{1} .
\end{aligned}
$$




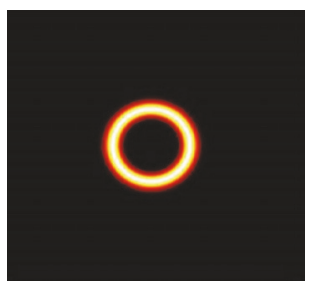

(a)

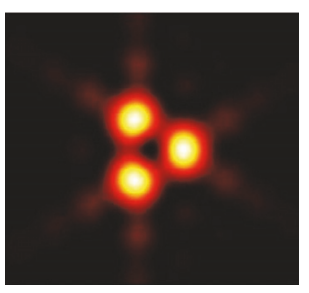

(f)

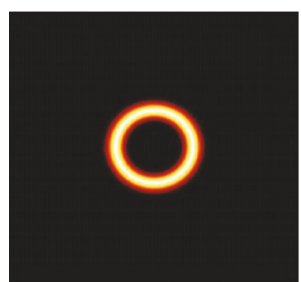

(b)

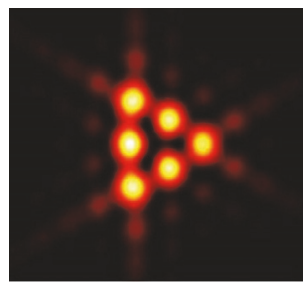

(g)

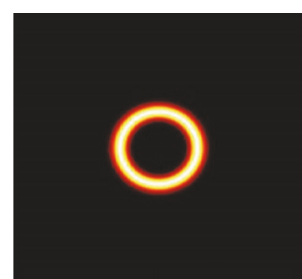

(c)

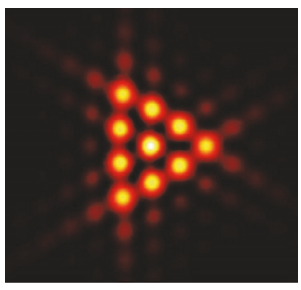

(h)

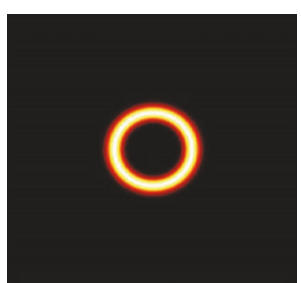

(d)

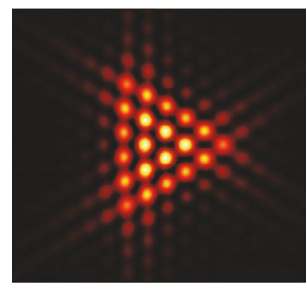

(i)

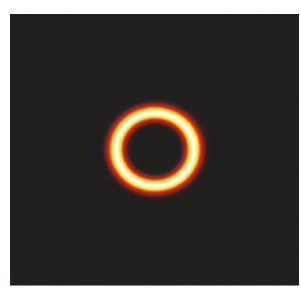

(e)

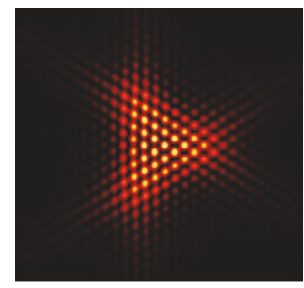

(j)

FIGURE 3: Simulated intensity patterns for POVs and their respective Fraunhofer diffraction by an equilateral triangular aperture. (a)-(e) POVs with topological charges $m=1,2,3,5$, and 10, respectively. (f)-(j) Truncated triangular lattices of POVs after the Fourier transformation of (a)-(e) for $P=1.3[\mathrm{~mm}]$.

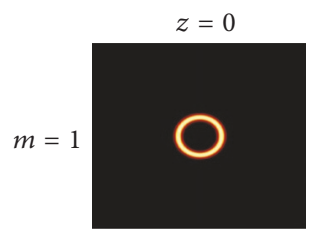

(a)

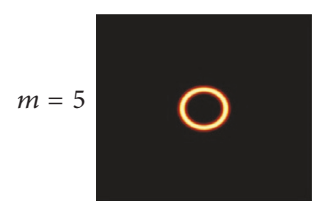

(g)

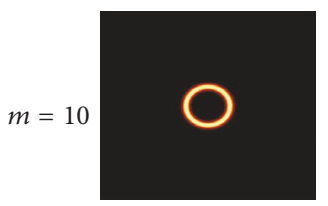

(m)

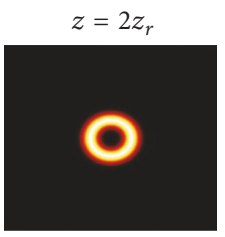

(b)

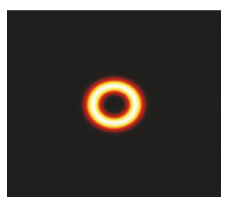

(h)

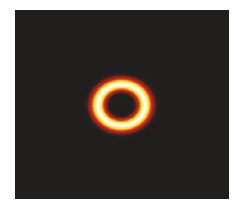

(n)

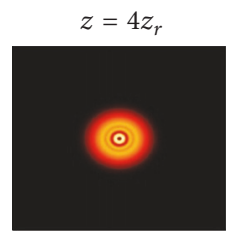

(c)

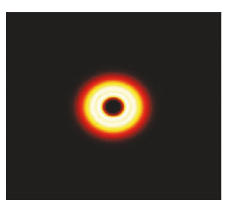

(i)

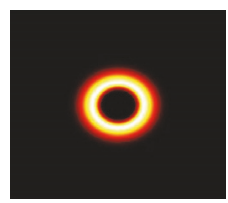

(o)

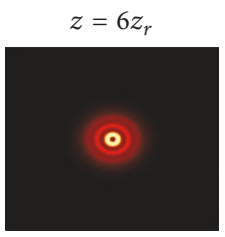

(d)

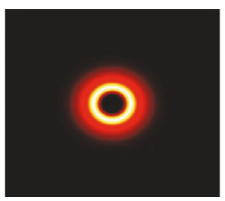

(j)

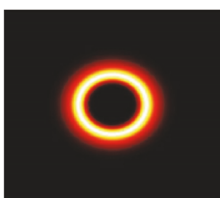

(p)

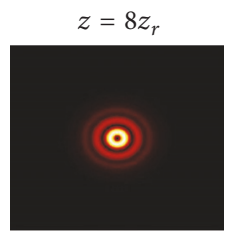

(e)

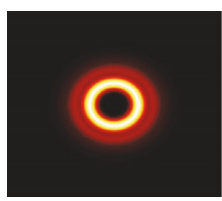

(k)

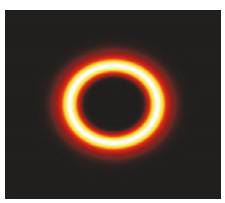

(q)

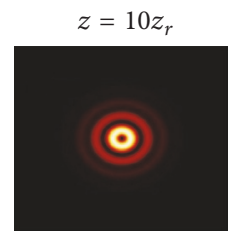

(f)

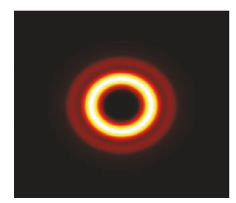

(l)

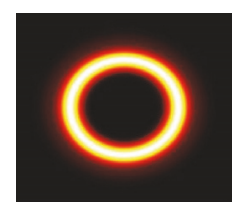

(r)

FIGURE 4: Simulated intensity patterns for POVs in free space propagation with different TC (rows) and same distance (columns). (a)-(f) POV with topological charge $m=1$; (g)-(l) POV with $m=5$; and (m)-(r) POV with $m=10$.

After inserting the expression of the amplitude field of a POV in the back focal plane (see (3)) and evaluating analytically the integral, we obtain the complex amplitude of a POV in free space propagation [31]

$$
\begin{gathered}
U\left(r_{1}, \phi_{1}, z\right)=i^{m-1} \frac{w}{w_{1}}(-1)^{m} I_{m}\left(\frac{2 r_{1} r_{o p} e^{i \psi}}{w w_{1}}\right) \\
\cdot e^{i(m \phi+k z+\psi)} e^{-i k\left(r_{1}^{2}+r_{o p}^{2}\right) / 2 R} e^{-\left(z_{r}^{2} r_{1}^{2}+z^{2} r_{o p}^{2}\right) / z_{r}^{2} w_{1}^{2}},
\end{gathered}
$$

with $w_{1}$ and $R$, the beam and curvature radii, respectively. $\psi$ is the Gouy phase of the Gaussian beam. These parameters are related to propagation distance $z$, waist size $w$, and the Rayleigh range $z_{r}$ by

$$
\begin{aligned}
w_{1} & =w \sqrt{1+\left(\frac{z}{z_{r}}\right)^{2}} ; \\
R & =\frac{z^{2}+z_{r}^{2}}{z} .
\end{aligned}
$$




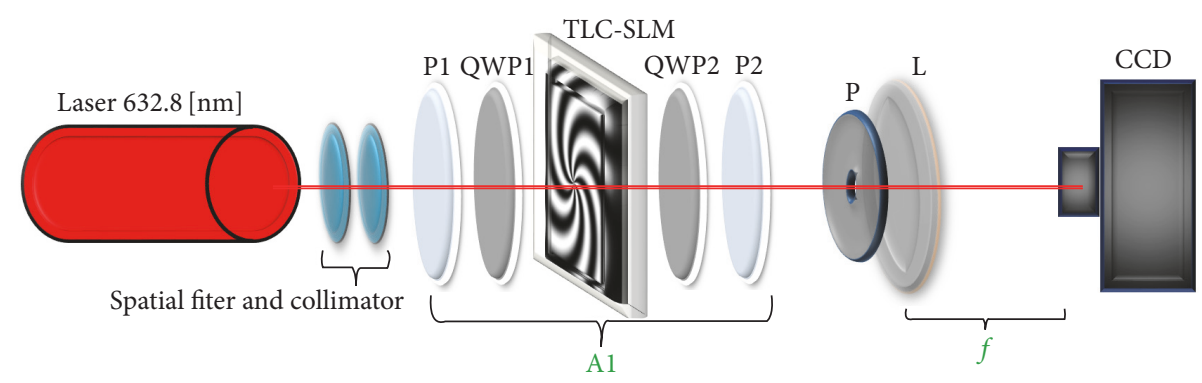

FIGURE 5: Schema of experimental setup for generated POVs: spatial filter and collimator; TLC-SLM: transmission liquid crystal spatial light modulator; P1: polarizer; QWP1: quarter-wave plate 1; P2: analyzer; QWP2: quarter-wave plate 2; P: pupil; L: Fourier lens with $f=380$ [mm] and CCD camera. The TLC-SLM displays an example of computerized hologram used to generate a POV.

$$
\begin{aligned}
\psi & =\tan ^{-1}\left(\frac{z}{z_{r}}\right) ; \\
z_{r} & =\frac{k w^{2}}{2} .
\end{aligned}
$$

Using (12) can simulate the Fresnel diffraction pattern field of a POV in free space propagation at different lengths. Figure 4 shows a collection of theoretical intensity distributions of the POVs with topological charges $m=1,5$, and 10 (different rows), at distances $z=0,2,4,6,8$ and $z=10$ times the Rayleigh range. As we can observe from each row in Figure 4, the POV intensity profile changes when distance increases. We can appreciate that only at $z=0$ the intensity distribution for topological charges $m=1, m=5$, and $m=10$ maintains the main POV characteristics: average ring-diameter and ring-width, independent of the topological charge. However, for other distances, the POV intensity distribution shows a drastic characteristics change; it develops other secondary doughnuts. Then, a POV in free space propagation has variant diffraction properties and we cannot called POV. This conclusion can be inferred from (12) due to field dependence with distance $z$ through combination of the exponential and modified Bessel functions.

\section{Experimental Setup}

Figure 5 shows the experimental setup for generating perfect optical vortices. A random polarized light from a HeliumNeon laser (spectra physics, power $2.5 \mathrm{~mW}$, and wavelength $632.8[\mathrm{~nm}])$ in the transverse electromagnetic ground state mode $\mathrm{TEM}_{00}$ is spatially filtered and collimated. Subsequently, the beam is directed toward an experimental configuration composed of two lineal polarizers, a transmission liquid crystal spatial light modulator (TLC-SLM, Sony model LCX038ARA spatial resolution: $1024(\mathrm{H}) \times 768(\mathrm{~V})$ pixels), and two quarter-wave plates. In order to create phase masks with combination of axicon and spiral functions, the TLCSLM has been placed in the arrangement Al composed by linear polarizer (P1), quarter-wave plate 1 (QWP1), TLC-SLM, linear analyzer (P2), and quarter-wave plate 2 (QWP2). In this arrangement the orientation of the linear polarizer, analyzer, and the waveplates in front of and behind the TLC-SLM was obtained through calibration previously of TLC-SLM in phase only modulation $[40,41]$ for $\lambda=632.8[\mathrm{~nm}]$. Our
TLC-SLM showed that the maximum phase modulation is $\theta_{\text {max }} \approx 1.3 \pi$, which corresponds to $N=3$ phase levels [42].

The BG beams with the same axicon period $d$, but different topological charge $m$, are generated using the phase computerized holograms. These computerized holograms displayed on the TLC-SLM are calculated through the following transmission function:

$$
\begin{aligned}
& T(x, y) \\
& =t_{0} \bmod [\operatorname{phase}\{\exp (i 2 \pi X)+\exp i \Gamma(x, y)\}, 1.3 \pi],
\end{aligned}
$$

where $t_{0}$ is the modulation depth, $X$ is the inverse of the spatial period, and $\Gamma(x, y)=\left(m \tan ^{-1}(y / x)+P \sqrt{x^{2}+y^{2}}\right)$ denotes the combination of axicon and spiral functions. The phase distribution is added modulo $1.3 \pi$ because this value is the maximum possible phase modulation for our TLC-SLM used. In addition, a linear phase shift (not shown in (14)) has been imposed onto incident laser beam by our TLC-SLM in order to separate the first order from the zero order beam (blazed hologram). The output BG beam after arrangement A1 is filtered from zero order using a pupil located just before the Fourier lens L. The filtered BG beam incident on Fourier lens (with 380 [mm] of focal length) allows obtaining the Fraunhofer field diffracted pattern. Finally, a CCD camera is placed at the lens back focal plane to detect and save the intensity distribution pattern of the optical field diffracted onto a computer hard drive.

For verifying the nature of the perfect optical vortex generated from Fourier transform of a Bessel-Gauss beam we used an equilateral triangular aperture placed at the back focal plane of L1 within the experimental setup shown in Figure 6. The diffracted field by the equilateral triangular aperture incident onto the lens L2, which is located at distance $f_{1}$ from the aperture, focuses the image onto CCD camera. With the lens L2, the far-field diffraction pattern in the back focal plane of L1 is imaged onto the CCD camera that records and saves the experimental images of the POV diffraction pattern. To avoid the effect of the quadratic phase factor on the experimental Fourier transform plane located onto the equilateral triangular aperture and to achieve the best Fourier transform, we need to adjust the distance between TLC-SLM and the lens L1 to $f$.

Figure 7 shows the experimental arrangement used for finding the POV diffraction intensity pattern in free space propagation after the back Fourier plane. Diffraction intensity 


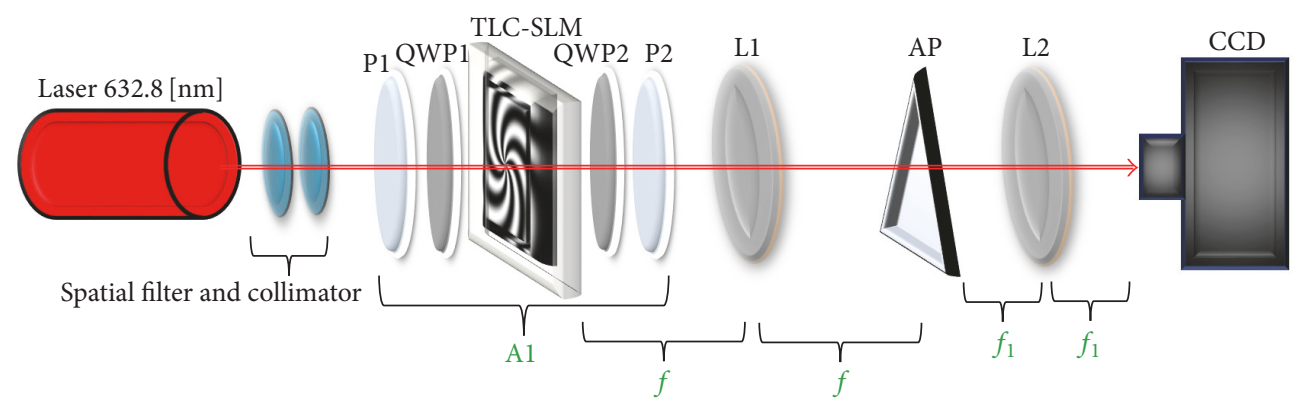

FIGURE 6: Schema of experimental setup for verifying the POV nature: spatial filter and collimator; TLC-SLM: transmission liquid crystal spatial light modulator; P1: polarizer; QWP1: quarter-wave plate 1; P2: analyzer; QWP2: quarter-wave plate 2; L1: Fourier lens 1; AP; triangular aperture; L2, Fourier lens 2 with $f_{1}=80$ [mm] and CCD camera. The TLC-SLM shows an example of computerized hologram used to generate a POV.

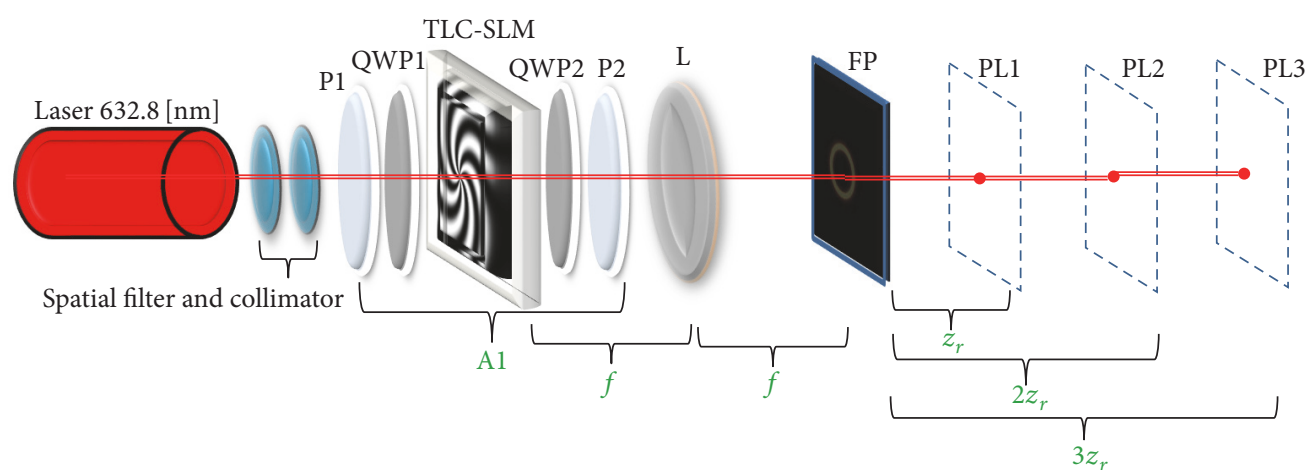

FIGURE 7: Schema of experimental setup for finding the POV diffraction intensity pattern after the Fourier plane: spatial filter and collimator; TLC-SLM: transmission liquid crystal spatial light modulator; P1: polarizer; QWP1: quarter-wave plate 1; P2: analyzer; QWP2: quarter-wave plate 2; L: Fourier lens; FP: Fourier plane; PL1: plane 1; PL2: plane 2; PL3: plane 3; $z_{r}$, Rayleigh range; and $f=380$ [mm]. The TLC-SLM shows an example of computerized hologram use to generate a POV.

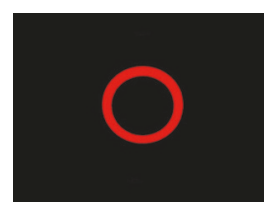

(a)

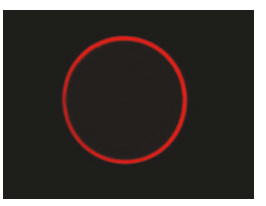

(f)

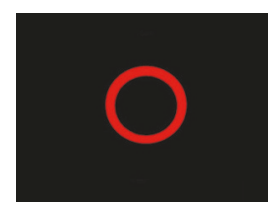

(b)

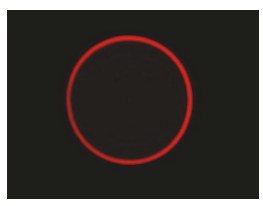

(g)

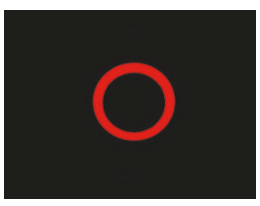

(c)

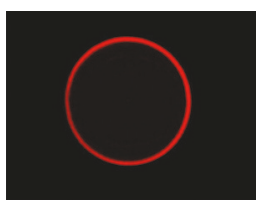

(h)

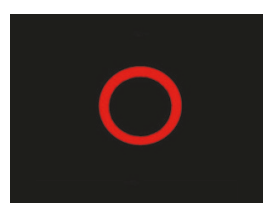

(d)

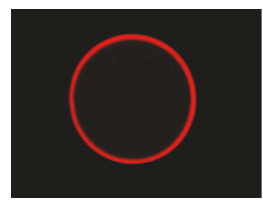

(i)

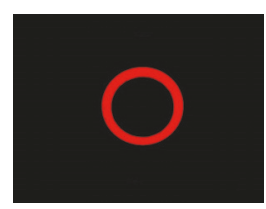

(e)

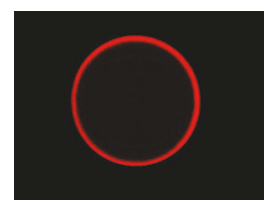

(j)

FIGURE 8: Experimental POVs intensity pattern for topological charges $m=1,2,3,5$, and 10, respectively. (a)-(e) For axicon period of $P=1.3$ $[\mathrm{mm}]$. (f)-(j) For axicon period of $P=0.7[\mathrm{~mm}]$.

patterns are recorded by a CCD camera placed after back focal plane of the Fourier lens L at positions PL1 $\left(z=z_{r}\right)$, PL2 $\left(z=2 z_{r}\right)$, and PL3 $\left(z=3 z_{r}\right)$. We have adjusted the distance between TLC-SLM and the lens L1 to $f$ for the purpose of canceling the quadratic phase factor in the Fourier spectrum.

\section{Experimental Results and Discussion}

Figure 8 shows the intensity distributions of the POV beams generated experimentally with topological charges $m=1,2$,
3,5 , and 10, for the phase masks with axicon period $P=$ $1.3[\mathrm{~mm}$ ] (Figures $8(\mathrm{a})-8(\mathrm{e})$ ) and $P=0.7$ [mm] (Figures $8(f)-8(j))$, using the experimental setup of Figure 6 .

In this figure, one can see by naked eye that the intensity distributions are invariant with the topological charge. One can also observe that size of the intensity distributions changes with the axicon period according to theory; that is, $P$ is inverse proportional to $r_{o p}$. All the images are considered in the same scale. Figures $9(f)-9(j)$ show experimental results of the vorticity verification of the POVs. In Figures 9(f)-9(j) 


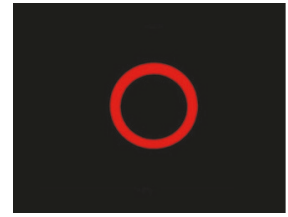

(a)

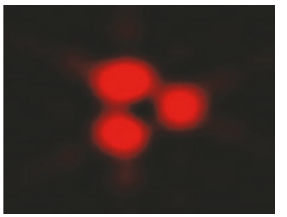

(f)

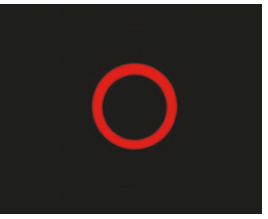

(b)

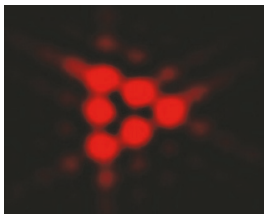

(g)

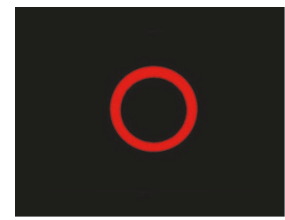

(c)

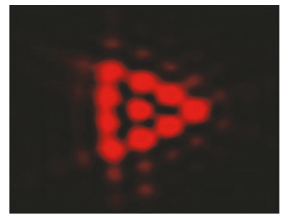

(h)

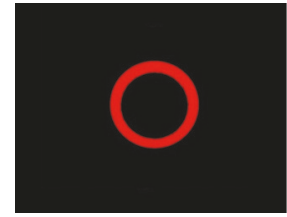

(d)

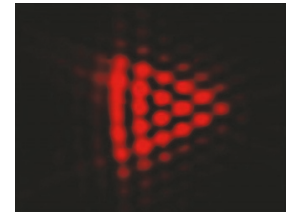

(i)

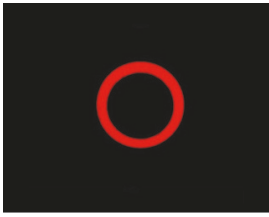

(e)

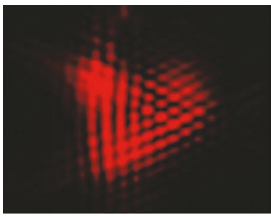

(j)

FIGURE 9: POVs intensity patterns and their respective experimental Fraunhofer diffraction. (a) $-(\mathrm{e})$ POVs with topological charges $m=$ $1,2,3,5$, and 10 for $P=1.3$ [mm], respectively. (f)-(j) Truncated triangular lattices of respective POVs after Fourier transformation.

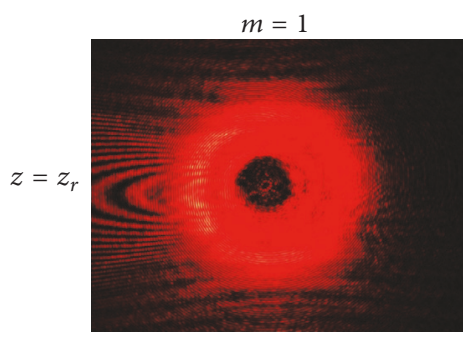

(a)

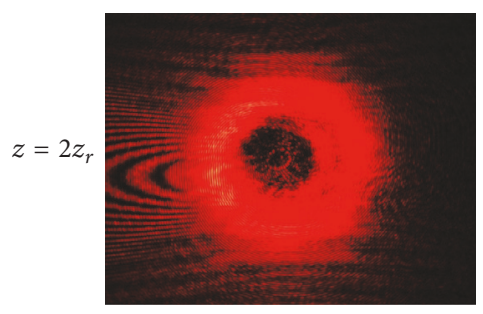

(d)

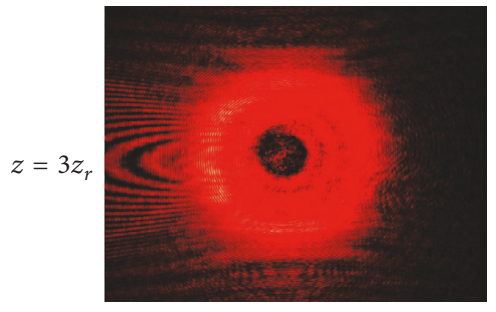

(g)

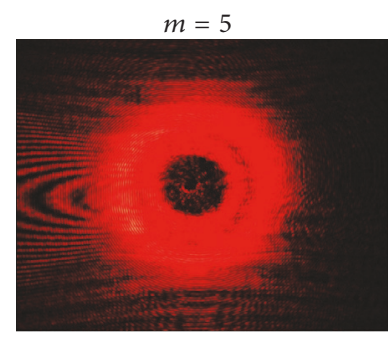

(b)

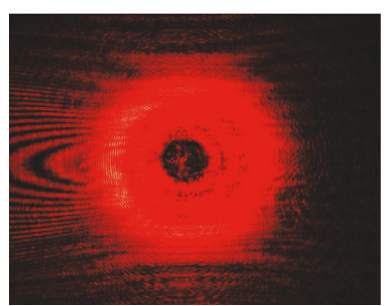

(e)

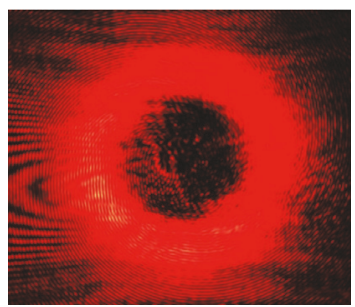

(h)

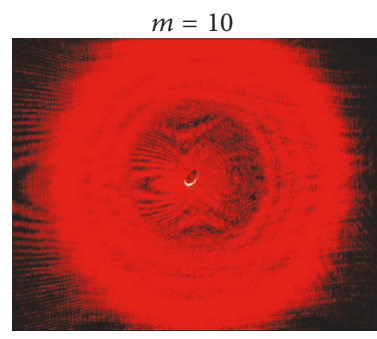

(c)

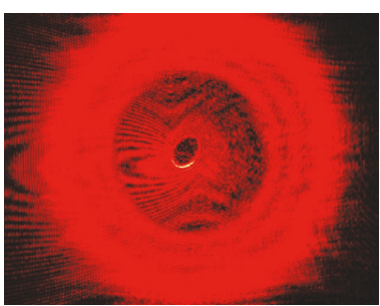

(f)

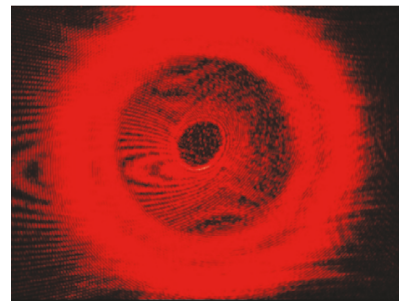

(i)

FIGURE 10: POV experimental intensity patterns in free space propagation with different topological charge (columns) at the same distance (rows). (a)-(c) POVs at distance $z_{r}$; (d)-(f) POVs at distance $2 z_{r}$; and (g)-(i) POVs at distance $3 z_{r}$.

we can note that the pattern of the Fraunhofer diffraction intensity distribution of a POV by an equilateral triangular aperture exhibits a truncated triangular lattice. The size of the triangular array and the number of spots grow with the increase of the topological charge. In addition, we can observe that the number of brilliant spots in any external side minus one equals the topological charge value of the POV.
These measurements have an excellent agreement with the theoretical results of Figure 2(b). For the topological charge $m=10$, the magnitude of the vorticity in the respective POV does not match well because this value is the limit of the measurements using an equilateral triangular aperture [43].

Finally, each column of Figure 10 exhibits the diffraction patterns captured by CCD camera at positions $z_{r}, 2 z_{r}$, and 
$3 z_{r}$ after the back Fourier plane of lens, when the POV with topological charges $m=1,5$, and 10 diffracts in free space, respectively. In these intensity patterns, we can see basically that the intensity distributions are size variant with the topological charge order. These experimental data clearly manifest the dependence between the free space diffraction pattern of a POV with a well-defined topological charge and their distance of propagation. In this figure, the POV in free space propagation covers the entire area of CCD camera at $z=3 z_{r}$, which did not allow us to record them at higher distances.

\section{Conclusions}

We proved that an optical perfect vortex could be essentially created by the Fourier transformation of an adequate combination of an axicon function and a spiral function. We also showed that the size of the annular vortex on the back Fourier plane of the transforming lens is independent of topological charge of spiral phase function. Finally, numerical and experimental results for the free space diffraction propagation of perfect optical vortex, after the back Fourier plane, show that their intensity pattern depends on topological charge and propagation distance.

\section{Conflicts of Interest}

The authors declare that they have no conflicts of interest.

\section{Acknowledgments}

Thanks are due to Universidad Industrial de Santander for the support of Research and Services Vice-Chancellor VIE Funding Projects 5191/5803 and 5708, both from the institutional program for consolidation of research groups, years 2012 and 2013, respectively. The authors also acknowledge support from Colciencias under Project 110256934957, "Optics Devices for Quantum Key Distribution, High Dimensionality Systems Based in Orbital Angular Momentum of Light." Funds were obtained from the National Call for the Bank of Projects in Science, Technology, and Innovation 2012.

\section{References}

[1] A. M. Yao and M. J. Padgett, "Orbital angular momentum: origins, behavior and applications," Advances in Optics and Photonics, vol. 3, no. 2, pp. 161-204, 2011.

[2] M. E. Ketara and E. Brasselet, "Observation of self-induced optical vortex precession," Physical Review Letters, vol. 110, no. 23, Article ID 233603, 2013.

[3] J. Leach, M. R. Dennis, J. Courtial, and M. J. Padgett, "Vortex knots in light," New Journal of Physics, vol. 7, article 055, 2005.

[4] L. Allen, M. W. Beijersbergen, R. J. C. Spreeuw, and J. P. Woerdman, "Orbital angular momentum of light and the transformation of Laguerre-Gaussian laser modes," Physical Review A, vol. 45, no. 11, pp. 8185-8189, 1992.

[5] J. E. Curtis and D. G. Grier, "Modulated optical vortices," Optics Letters, vol. 28, no. 11, pp. 872-874, 2003.
[6] A. Jesacher, S. Fürhapter, C. Maurer, S. Bernet, and M. RitschMarte, "Holographic optical tweezers for object manipulations at an air-liquid surface," Optics Express, vol. 14, no. 13, pp. 63426352,2006

[7] A. Arias, S. Etcheverry, P. Solano et al., "Simultaneous rotation, orientation and displacement control of birefringent microparticles in holographic optical tweezers," Optics Express, vol. 21, no. 1, pp. 102-111, 2013.

[8] H. Wang, Z. Xie, M. Zhang et al., "A miniaturized optical fiber microphone with concentric nanorings grating and microsprings structured diaphragm," Optics and Laser Technology, vol. 78, pp. 110-115, 2016.

[9] K. Crabtree, J. A. Davis, and I. Moreno, "Optical processing with vortex-producing lenses," Applied Optics, vol. 43, no. 6, pp. 1360-1367, 2004.

[10] T. Ehmke, T. H. Nitzsche, A. Knebl, and A. Heisterkamp, "Molecular orientation sensitive second harmonic microscopy by radially and azimuthally polarized light," Biomedical Optics Express, vol. 5, no. 7, pp. 2231-2246, 2014.

[11] A. Popiołek-Masajada, B. Sokolenko, I. Augustyniak, J. Masajada, A. Khoroshun, and M. Bacia, "Optical Vortex Scanning in an aperture limited system," Optics and Lasers in Engineering, vol. 55, pp. 105-112, 2014.

[12] I. B. Djordjevic and M. Arabaci, "LDPC-coded orbital angular momentum (OAM) modulation for free-space optical communication," Optics Express, vol. 18, no. 24, pp. 24722-24728, 2010.

[13] Y. Yan, G. Xie, M. P. J. Lavery et al., "High-capacity millimetrewave communications with orbital angular momentum multiplexing," Nature Communications, vol. 5, article 4876, 2014.

[14] H. Huang, G. Xie, Y. Yan et al., "100 Tbit/s free-space data link enabled by three-dimensional multiplexing of orbital angular momentum, polarization, and wavelength," Optics Letters, vol. 39, no. 2, pp. 197-200, 2014.

[15] N. Bozinovic, Y. Yue, Y. Ren et al., “Terabit-scale orbital angular momentum mode division multiplexing in fibers," Science, vol. 340, no. 6140, pp. 1545-1548, 2013.

[16] C. Brunet, P. Vaity, Y. Messaddeq, S. LaRochelle, and L. A. Rusch, "Design, fabrication and validation of an OAM fiber supporting 36 states," Optics Express, vol. 22, no. 21, pp. 2611726127, 2014.

[17] C.-S. Guo, X. Liu, J.-L. He, and H.-T. Wang, "Optimal annulus structures of optical vortices," Optics Express, vol. 12, no. 19, pp. 4625-4634, 2004.

[18] V. Arrizón, S. Chávez-Cerda, U. Ruiz, and R. Carrada, "Periodic and quasi-periodic non-diffracting wave fields generated by superposition of multiple Bessel beams," Optics Express, vol. 15, no. 25, pp. 16748-16753, 2007.

[19] R. Vasilyeu, A. Dudley, N. Khilo, and A. Forbes, "Generating superpositions of higher-order Bessel beams," Optics Express, vol. 17, no. 26, pp. 23389-23395, 2009.

[20] J. Chen, X. Zhao, Z. Fang, S. Zhu, and X.-C. Yuan, "Explicit relations and optimal parameters for sidelobe suppression in optical vortices with a modified Bessel function," Journal of the Optical Society of America A: Optics and Image Science, and Vision, vol. 27, no. 4, pp. 935-940, 2010.

[21] J. Chen, Y. Yu, and F. Wang, "Production of annular flat-topped vortex beams," Chinese Optics Letters, vol. 9, no. 1, Article ID 011402, 2011.

[22] A. Calatayud, J. A. Rodrigo, L. Remón, W. D. Furlan, G. Cristóbal, and J. A. Monsoriu, "Experimental generation and characterization of Devil's vortex-lenses," Applied Physics B: Lasers and Optics, vol. 106, no. 4, pp. 915-919, 2012. 
[23] G. Campbell, B. Hage, B. Buchler, and P. K. Lam, "Generation of high-order optical vortices using directly machined spiral phase mirrors," Applied Optics, vol. 51, no. 7, pp. 873-876, 2012.

[24] E. Rueda, D. Muñetón, J. A. Gómez, and A. Lencina, "Highquality optical vortex-beam generation by using a multilevel vortex-producing lens," Optics Letters, vol. 38, no. 19, pp. 39413944, 2013.

[25] Z. Li and C. Cheng, "Generation of second-order vortex arrays with six-pinhole interferometers under plane wave illumination," Applied Optics, no. 8, pp. 1629-1635, 2014.

[26] Y. Chen, Z.-X. Fang, Y.-X. Ren, L. Gong, and R.-D. Lu, "Subwavelength grating based metal-oxide nano-hair structures for optical vortex generation," Optics Express, vol. 23, no. 15, pp. 19056-19065, 2015.

[27] X. Zhang, A. Wang, R. Chen, Y. Zhou, H. Ming, and Q. Zhan, "Generation and conversion of higher order optical vortices in optical fiber with helical fiber bragg gratings," Journal of Lightwave Technology, vol. 34, no. 10, pp. 2413-2418, 2016.

[28] A. Ostrovsk, C. Rickenstor-Parrao, and V. Arrizón, “Generation of the 'perfect' optical vortex using a liquid-crystal spatial light modulator," Optics Letters, vol. 38, no. 4, pp. 534-536, 2013.

[29] J. García-García, C. Rickenstorff-Parrao, R. Ramos-García, V. Arrizón, and A. S. Ostrovsky, "Simple technique for generating the perfect optical vortex," Optics Letters, vol. 39, no. 18, pp. 5305-5308, 2014.

[30] M. Chen, M. Mazilu, Y. Arita, E. M. Wright, and K. Dholakia, "Dynamics of microparticles trapped in a perfect vortex beam," Optics Letters, vol. 38, no. 22, pp. 4919-4922, 2013.

[31] P. Vaity and L. Rusch, "Perfect vortex beam: Fourier transformation of a Bessel beam," Optics Letters, vol. 40, no. 4, pp. 597-600, 2015.

[32] J. Yu, C. Zhou, Y. Lu, J. Wu, L. Zhu, and W. Jia, "Square lattices of quasi-perfect optical vortices generated by two-dimensional encoding continuous-phase gratings," Optics Letters, vol. 40, no. 11, pp. 2513-2516, 2015.

[33] F. Gori, G. Guattari, and C. Padovani, "Bessel-Gauss beams," Optics Communications, vol. 64, no. 6, pp. 491-495, 1987.

[34] M. Abramowitz and I. Stegun, Handbook of Mathematical Functions, Dover Books on Mathematics, Washington, DC, USA, 9th edition, 1964.

[35] J. Durnin, "Exact solutions for nondiffracting beams. I. The scalar theory," Journal of the Optical Society of America A: Optics and Image Science, and Vision, vol. 4, no. 4, pp. 651-654, 1987.

[36] J. Durnin, J. Miceli Jr., and J. H. Eberly, "Diffraction-free beams," Physical Review Letters, vol. 58, no. 15, pp. 1499-1501, 1987.

[37] R. C. Smith and J. S. Marsh, "Diffraction patterns of simple apertures," Journal of the Optical Society America, vol. 64, no. 6, pp. 798-803, 1974.

[38] J. M. Hickmann, E. J. S. Fonseca, W. C. Soares, and S. ChávezCerda, "Unveiling a truncated optical lattice associated with a triangular aperture using light's orbital angular momentum," Physical Review Letters, vol. 105, no. 5, Article ID 053904, 2010.

[39] J. Goodman, Introduction to Fourier Optics, McGraw-Hill, New York, NY, USA, 2nd edition, 1996.

[40] J. A. Davis, I. Moreno, and P. Tsai, "Polarization eigenstates for twisted-nematic liquid-crystal displays," Applied Optics, vol. 37, no. 5, pp. 937-945, 1998.

[41] I. Moreno, P. Velásquez, C. R. Fernández-Pousa, M. M. SánchezLópez, and F. Mateos, "Jones matrix method for predicting and optimizing the optical modulation properties of a liquid-crystal display," Journal of Applied Physics, vol. 94, no. 6, pp. 3697-3702, 2003.
[42] V. V. Kotlyar and A. A. Kovalev, "Fraunhofer diffraction of the plane wave by a multilevel (quantized) spiral phase plate," Optics Letters, vol. 33, no. 2, pp. 189-191, 2008.

[43] J. G. Silva, A. J. Jesus-Silva, M. A. R. C. Alencar, J. M. Hickmann, and E. J. S. Fonseca, "Unveiling square and triangular optical lattices: a comparative study," Optics Letters, vol. 39, no. 4, pp. 949-952, 2014. 

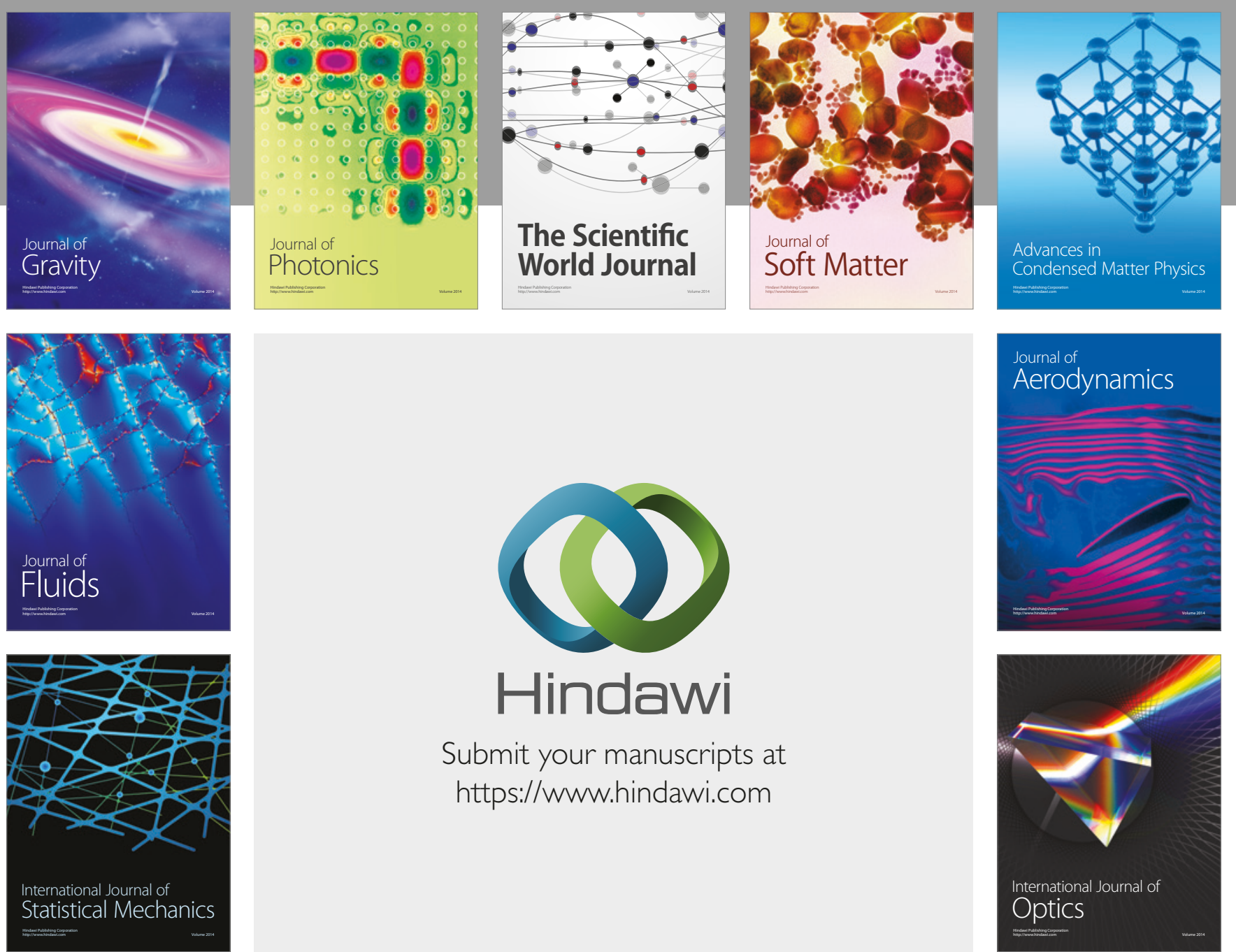

Submit your manuscripts at

https://www.hindawi.com
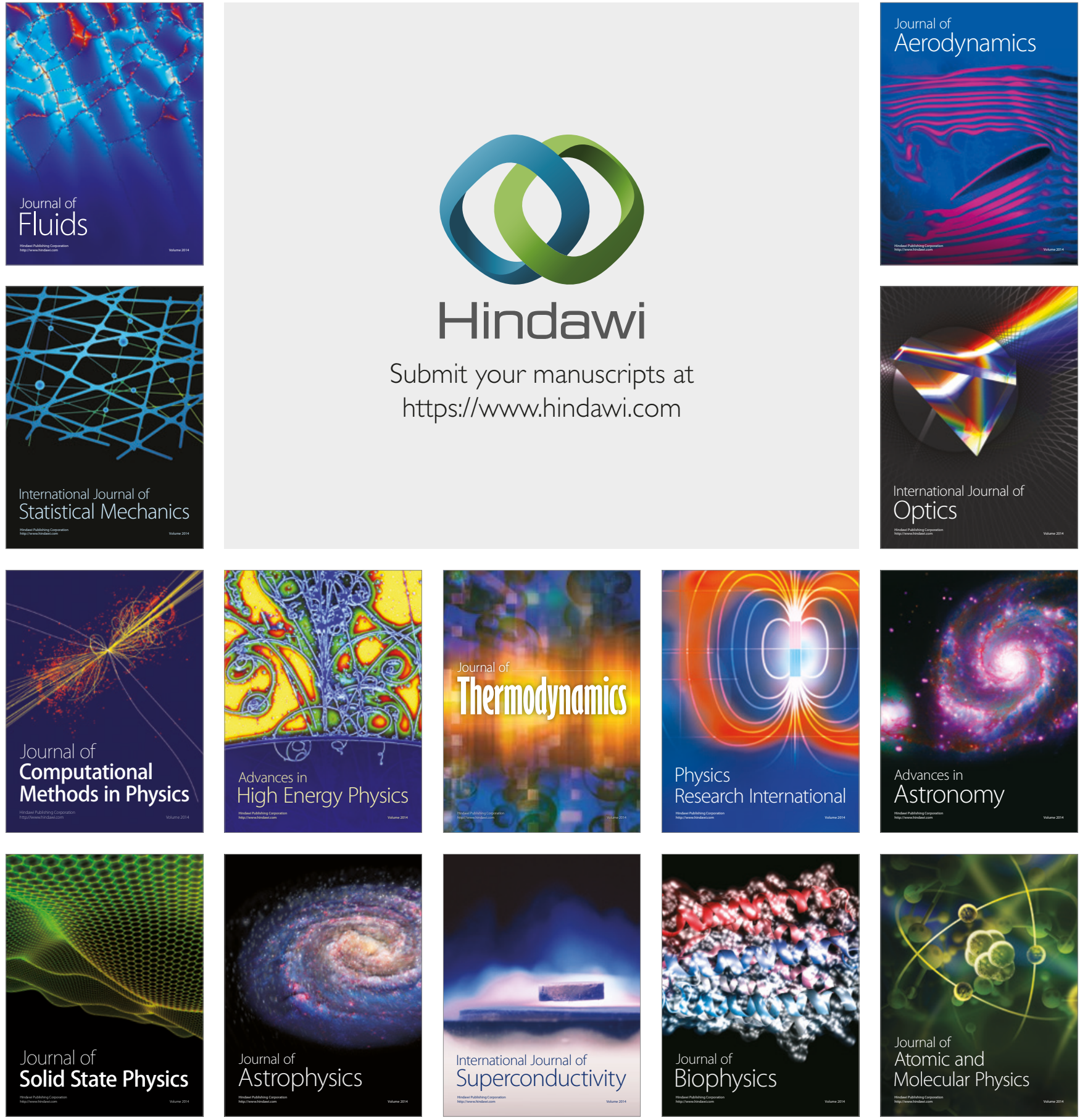